Merci d'utiliser le titre suivant lorsque vous citez ce document :

M. Alston, J. (2010-08-01), « Les avantages de la recherchedéveloppement, de l'innovation et de l'accroissement de la productivité dans le secteur agricole », Éditions OCDE, Paris. http://dx.doi.org/10.1787/5km91nfjnhq3-fr

\title{
Les avantages de la
} recherche-développement, de l'innovation et de l'accroissement de la productivité dans le secteur agricole

\author{
Julian M. Alston
}

La version originale de ce document a été publiée comme suit :

M. Alston, J. (2010-08-01), "The Benefits from Agricultural Research and Development, Innovation, and Productivity Growth", OECD Food, Agriculture and Fisheries Papers, No. 31, OECD Publishing, Paris. http://dx.doi.org/10.1787/5km91nfsnkwg-en 


\title{
LES AVANTAGES DE LA RECHERCHE-DÉVELOPPEMENT, DE L'INNOVATION ET DE L'ACCROISSEMENT DE LA PRODUCTIVITÉ DANS LE SECTEUR AGRICOLE
}

\author{
Julian M. Alston*
}

* Department of Agricultural and Resource Economics de l'Université de Californie, Davis. 


\section{Résumé}

Ce rapport examine la bibliographie sur le rôle que joue la recherchedéveloppement en agriculture en matière d'encouragement de l'innovation et de la productivité dans le secteur agricole. Cet examen cherche à clarifier les concepts et la terminologie utilisés dans le domaine, à donner une évaluation critique des méthodes rencontrées dans les études publiées, à rendre compte des principaux résultats et à en tirer des conclusions. Une constatation essentielle est que le taux de rentabilité, pour la collectivité, des investissements dans la recherche-développement en agriculture est généralement élevé. Les résultats précis varient en fonction des méthodes et des hypothèses de modélisation, en particulier des hypothèses concernant la distribution des retards, la nature de l'évolution technologique suscitée par la recherche et la nature des marchés des produits agricoles touchés.

Mots clés : Recherche-développement en agriculture, innovation, productivité agricole, croissance de la productivité. 


\section{Table des matières}

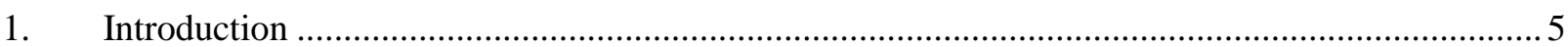

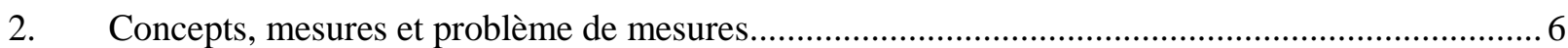

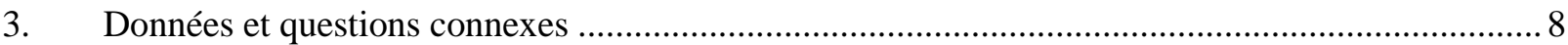

4. Problèmes d'attribution dans les modèles concernant les impacts de la recherche .........................9

5. Résultats sur les conséquences économiques de la recherche-développement en

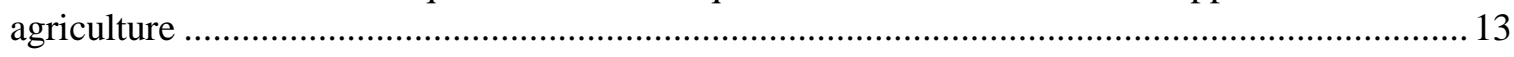

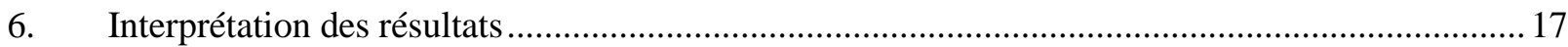

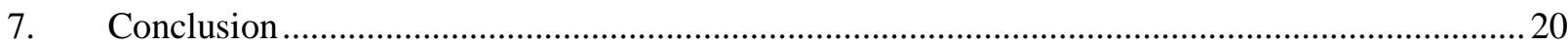

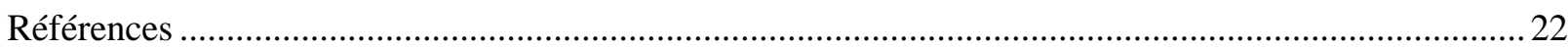





\section{Les avantages de la recherche-développement, de l'innovation et de l'accroissement de la productivité dans le secteur agricole}

\section{Introduction}

Au cours du demi-siècle passé environ, des centaines d'études ont été publiées pour rendre compte de mesures de la productivité agricole, des effets de la recherchedéveloppement (R-D) en agriculture sur l'innovation agricole et les courbes de productivité, ainsi que des résultats, pour la collectivité, des investissements dans la R-D en agriculture. Ce rapport résume et interprète les principaux résultats de ce corpus de travaux. L'accent est mis dans une certaine mesure sur les conséquences pouvant découler des différentes méthodes utilisées par les économistes sur leurs résultats concernant les impacts de la recherche.

L'examen se fonde sur plusieurs travaux récents qui traitent de ces mêmes questions de manière plus exhaustive. Les références comprennent tout spécialement :

- une méta-analyse des études de rentabilité de la recherche par Alston, Chan-Kang, Marra, Pardey et Wyatt (2000a), publiée sous une forme résumée par Alston, Marra, Pardey et Wyatt (2000b) ;

- une étude des publications sur l'innovation dans le secteur agricole par Pardey, Alston et Ruttan (2010), parue dans Handbook of Economics of Technical Change ;

- un ouvrage récent de Alston, Andersen, Pardey et James (2010), qui décrit la R-D et la productivité dans le secteur agricole aux États-Unis, examine les concepts et les mesures, et rend compte de nouvelles estimations de la rentabilité des investissements publics dans la R-D en agriculture aux États-Unis à l'aide de données au niveau des États ;

- une étude des publications sur l'économie de la R-D en agriculture par Alston, Pardey, James and Andersen (2009), parue dans Annual Review of Resource Economics; et

- des travaux récents de Alston, Beddow et Pardey $(2008,2009)$ sur les schémas mondiaux de productivité agricole, qui figurent avec un certain nombre d'études par pays dans un ouvrage publié par Alston, Babcock et Pardey (2010).

Le lecteur trouvera dans ces publications des détails plus complets sur les questions abordées et les résultats, ainsi qu'une liste plus complète des études sur lesquelles s'appuie ce rapport. 


\section{Concepts, mesures et problème de mesures}

Les économistes agricoles utilisent des modèles d'offre et de demande des marchés des produits de base pour représenter les effets de la recherche en agriculture. Il y eut d'abord Schultz (1953) et Griliches (1958), puis vinrent Petersen (1967), Duncan et Tisdell (1971), Duncan (1972), Akino et Hayami (1975), et Scobie (1976), entre autres. Ce genre de modèle est utilisé explicitement dans un grand nombre d'études. Il est implicite dans d'autres études qui déduisent un taux de rentabilité de la recherche à partir des paramètres d'un modèle économétrique de la production (par exemple Evenson, 1967) ou bien utilisent des approximations raccourcies pour mesurer les avantages (par exemple Griliches, 1958).

Dans le modèle standard des avantages de la recherche tel qu'il est développé par exemple dans l'ouvrage de Alston, Norton et Pardey (1995), la recherche occasionne une réorientation à la baisse de la courbe de l'offre de produits agricoles par rapport à la courbe stationnaire de la demande, ce qui engendre une augmentation des quantités produites et consommées et en abaisse le prix. Les avantages sont évalués au moyen de mesures marshalliennes des variations du surplus du consommateur suscitées par la recherche, pour ce qui est des avantages du consommateur, et des variations du surplus du producteur suscitées par la recherche, pour ce qui est des avantages du producteur. Les avantages annuels totaux bruts de la recherche (GARB) dépendent essentiellement de l'ampleur du déplacement de l'offre suscité par la recherche (variable dans le temps et exprimé sous la forme d'un déplacement vertical d'une amplitude égale à une proportion, $k$, du prix initial) et de la taille du secteur auquel il s'applique ${ }^{1}$. En effet, une approximation courante introduite par Griliches (1958) est $G A R B=k P Q$, où $P$ est le prix du produit agricole et $Q$ est la quantité annuelle à laquelle s'applique le déplacement de l'offre. D'autres aspects de l'analyse ont généralement des effets de second ordre sur les mesures des avantages totaux, mais peuvent avoir des conséquences importantes pour la distribution des avantages entre les producteurs et les consommateurs et autres agents ${ }^{2}$.

Certains points abordés dans les publications concernent les méthodes utilisées pour mesurer le principal facteur qui détermine les avantages totaux mesurés, à savoir la diminution, imputable à la recherche, du coût unitaire de production à l'échelle du secteur telle qu'elle est représentée par le déplacement de l'offre, $k$ (par exemple, à partir de taux d'adoption conjugués à des variations des rendements expérimentaux ou des rendements

1. Comme l'ont fait remarquer Alston, Norton et Pardey (1995, pp. 60-61), et comme cela a été plus récemment précisé par Oehmke et Crawford (2002), l'élasticité de l'offre peut avoir des conséquences importantes pour les mesures des avantages de la recherche si elle est utilisée pour convertir un déplacement supposé horizontal en déplacement vertical, ou inversement.

2. La distribution des avantages entre producteurs et consommateurs dépend des élasticités relatives de l'offre et de la demande, de la nature du déplacement de l'offre suscité par la recherche et, ce qui est moins important, des formes fonctionnelles de l'offre et de la demande (Alston, Norton et Pardey, 1995, examinent ces questions). La nature du déplacement de l'offre suscité par la recherche est controversée parce qu'elle est importante, en particulier pour les résultats concernant la distribution des avantages, et parce qu'elle n'est pas facile à observer. La distribution des avantages des producteurs entre eux est problématique elle aussi. Même si nous pouvons être assurés qu'une nouvelle technologie peut être profitable à l'ensemble des producteurs, ceux qui ne l'adoptent pas n'en retirent pas d'avantage et risquent même de se retrouver dans une situation plus défavorable si son adoption par d'autres entraîne des baisses de prix. 
commerciaux, ou à partir de variations de la productivité totale des facteurs). Ce choix est souvent régi par l'axe général de l'analyse (par exemple, l'évaluation des avantages du développement d'une amélioration variétale particulière ou l'évaluation d'un système national de recherche agricole, qu'elles soient réalisées ex ante ou a posteriori), ainsi que par la disponibilité des données et autres informations.

La mesure de l'ampleur et de la répartition des avantages de la recherche subit les effets de diverses complications qui peuvent être prises en compte dans le modèle de base pour l'étoffer. L'introduction du commerce international est un perfectionnement classique du modèle simple, grâce auquel nous pouvons mesurer des impacts sur le bienêtre pour différents agrégats spatiaux ou groupes de marchés. Les choses deviennent légèrement plus compliquées lorsque nous admettons dans le même modèle les retombées technologiques. Des modèles multimarchés plus complexes et plus élaborés sont nécessaires pour décomposer verticalement la structure du marché, afin de représenter différentes étapes de la chaîne de commercialisation, ou horizontalement, afin de représenter différents marchés géopolitiques ou spatiaux pour un produit donné ou différents produits (voire différentes qualités d'un même produit). Alston, Norton et Pardey (1995) établissent les fondements théoriques de ces méthodes et plusieurs études rendent compte d'applications spécifiques ${ }^{3}$.

Il est possible également, pour affiner le modèle de base, de ne pas le circonscrire à la R-D publique et aux seules distorsions des marchés imputables à celle-ci. Le modèle de base table sur l'hypothèse que les résultats de la recherche sont donnés gratuitement. Les modèles qui prennent en considération les technologies exclusives (par exemple Moschini et Lapan, 1997, et d'autres) n'ont pas été beaucoup employés jusqu'à maintenant dans les travaux appliqués et nous disposons de très peu d'éléments sur la distribution des avantages de la recherche privée entre les concepteurs et fournisseurs de technologies et les autres agents, notamment les agriculteurs, les consommateurs et l'agro-industrie. Le modèle de base part aussi du principe que le marché des produits agricoles est concurrentiel et qu'il ne connaît pas d'autre distorsion. Des modèles des avantages de la recherche ont été étendus afin d'y inclure différents types de distorsions du marché et notamment (a) celles qui découlent de mesures gouvernementales comme les programmes liés à des produits agricoles ou les obstacles aux échanges (par exemple, Alston, Edwards et Freebairn, 1988) y compris l'absence de taxes optimales sur les échanges dans le cas d'un grand pays (par exemple Alston et Martin, 1995) ; (b) celles qui résultent de l'exercice d'un pouvoir de marché par les intermédiaires (par exemple Huang et Sexton, 1996) ; et (c) celles qui découlent d'externalités environnementales (par exemple Antle et Pingali, 1994). En général, le résultat est que les distorsions ont dans ce contexte pour principal effet de modifier la distribution des avantages de la recherche, et qu'elles ont en comparaison peu d'incidences sur l'ampleur totale des avantages. Les autres types d'extensions du modèle de base destinées à tenir compte de la multiplicité des marchés ou de technologies exclusives donnent des résultats similaires. Comme le montre la méta-analyse conduite par Alston et al. (2000), la plupart des études qui rendent compte des taux de rentabilité de la R-D en agriculture définissent les avantages dans des termes relativement simples et ne traitent pas expressément les subtilités susceptibles d'influencer les avantages totaux dans leur ampleur mais qui, surtout, rejaillissent sur leur distribution.

3. Parmi les nombreux exemples, citons Mullen, Alston et Wohlgenant (1990), Freebairn (1992), Frisvold (1997), Wohglenant (1997), Davis et Espinoza (1998) ou Zhao et al. (2000). 


\section{Données et questions connexes}

Selon Gardner (1992) et Griliches (1994), la création de «données » donne lieu à beaucoup d'efforts et à des appréciations subjectives mais, bien souvent, les utilisateurs ne sont pas aussi informés qu'ils devraient l'être sur la façon dont les " données » ont été élaborées et sur la manière de les interpréter. En particulier, les données chiffrées sur les intrants agricoles (le capital, en particulier), la production et la productivité résultent en très grande partie de conversions opérées à partir d'un matériau brut. Étudier la rentabilité de la R-D en agriculture nécessite en outre de remanier sensiblement les données sur les investissements dans la recherche et la productivité de celle-ci alors qu'elles comportent déjà une grande part de subjectivité, dont une bonne partie n'est pas toujours visible pour l'utilisateur mais peut avoir des incidences importantes sur les résultats des études. Les limites imputables à la nature des données disponibles et à leur quantité, conjuguées aux méprises sur le sens des chiffrages ou à leur mauvaise utilisation pour d'autres raisons, contribuent probablement aux lacunes de certaines études sur le lien entre R-D et productivité dans l'agriculture. Certains de ces problèmes peuvent être insurmontables. D'autres peuvent être en partie résolus, à condition d'apporter un plus grand soin à l'analyse et à la présentation des résultats.

Dans de nombreux pays, on ne dispose que de données très limitées et d'une documentation incomplète sur les variables représentatives qui, très certainement, ne correspondent pas bien aux concepts concernés. Par exemple, beaucoup d'études s'appuient sur des données de la FAO sur la production et les intrants agricoles dont le champ est incomplet et qui ne permettent pas de créer des indices valables. Cependant, certains pays ont fait des progrès dans l'élaboration et la description des indicateurs de l'utilisation des intrants, de la production et de la productivité agricoles, et des investissements dans la recherche agricole, qui sont utilisés dans les études sur la rentabilité de la R-D en agriculture. Il reste toutefois des problèmes, comme le montre le cas des États-Unis ${ }^{4}$. Deux différents projets de recherche de longue haleine, l'un mené par Eldon Ball à l'ERS du ministère de l'Agriculture des États-Unis et l'autre par Philip Pardey à l'Université du Minnesota, ont abouti à l'établissement de séries de données dissemblables au niveau des États, lesquelles conduisent à des résultats sensiblement discordants en dépit d'objectifs fondamentalement communs, de l'utilisation d'une théorie appropriée sur les indices dans chacun des cas et d'informations de base similaires. (Pour plus de détails et des commentaires, voir Acquaye, Alston et Pardey, 2002 ; Andersen, 2005 ; Andersen, Alston et Pardey, 2008 ; Alston, Anderson, James et Pardey, 2010).

Il ressort des publications que, par rapport aux indicateurs de la productivité et de ses éléments, les indicateurs de l'investissement dans la recherche (et les mesures équivalentes des stocks de connaissances scientifiques) suscitent beaucoup moins d'efforts et d'attention. Ce désintérêt pourrait être relativement dommageable. Établir des

4. Andersen (2005) a dépouillé les études des schémas de productivité agricole aux États-Unis et décrit l'évolution des méthodes et des résultats. Parmi les études récentes de la productivité agricole aux États-Unis, citons les suivantes : Ball (1985), Evenson, Landau et Ballou (1987), Capalbo et Vo (1988), Craig et Pardey (1990a, 1990b et 2001), Jorgenson et Gollop (1992), Huffman et Evenson (1992, 1993), Ball (1994), Pardey, Craig et Deininger (1994), Ahearn et al. (1998), Ball et al. (1997), Ball et Nehring (1998), Ball et al. (1999), Acquaye, Alston et Pardey (2002), Alston, Andersen, James et Pardey (2010), Alston, Beddow et Pardey (2009). 
indicateurs des investissements dans la recherche agricole demande beaucoup de travail. Dans la plupart des pays, aucun organisme officiel ne publie d'indicateurs appropriés des investissements publics dans la recherche agricole sous une forme adéquate et sur une période suffisamment longue 5 . Pour mesurer correctement les dépenses publiques de recherche, il faut fouiller dans divers documents officiels et extraire des différentes lignes de dépenses les éléments qui concernent vraiment la recherche et qui s'appliquent vraiment à l'agriculture ; il faut faire des allers-retours d'un lieu à l'autre et dans le temps, et faire face aux modifications des définitions et des procédures de notification, ainsi qu'aux inévitables omissions.

Étant donné le temps qui s'écoule entre la R-D et ses applications dans l'agriculture, les études économétriques des séries chronologiques exigent de nombreuses années de données tant sur les investissements en R-D que sur la productivité. Un grand nombre d'études sont rendues difficiles par le manque de séries chronologiques suffisamment longues et ont recours à des artifices d'estimation qui faussent très vraisemblablement les résultats (restriction de la durée et de la forme de la distribution des décalages dans le temps ou estimation des données du passé à partir d'extrapolations directes du présent, une démarche qui n'apparaît pas toujours au lecteur dans la synthèse des résultats de la recherche). Les données sur les investissements privés dans la recherche sont particulièrement difficiles à obtenir, y compris sous la forme de séries chronologiques relativement courtes, puisque ces informations sont confidentielles - mêmes les sociétés faisant appel à l'épargne publique ne sont pas obligées de publier dans leurs rapports annuels des informations qui pourraient être utiles aux économistes ${ }^{6}$.

\section{Problèmes d'attribution dans les modèles concernant les impacts de la recherche}

Les problèmes d'attribution sur lesquels se sont penchés Alston et Pardey (2001) perturbent les études des effets de la recherche sur la productivité agricole. Les deux principaux domaines où l'on rencontre des difficultés sont (a) l'identification de l'élément de l'accroissement de productivité qui est attribuable à l'évolution des connaissances suscitée par la recherche, puis l'attribution de la responsabilité parmi les différents prestataires publics et privés de services de R-D (problème de l'attribution spatiale et institutionnalo-sectorielle), et (b) l'identification de la structure des retards, en l'occurrence des décalages entre la recherche et ses retombées (problème de l'attribution temporelle). Que l'analyse se concentre sur une innovation particulière ou qu'elle porte sur toutes les recherches entreprises par un système national, les problèmes sont globalement similaires; ils diffèrent toutefois dans le détail, et leur gravité est variable. Nombreuses sont les études qui tablent implicitement ou explicitement sur l'hypothèse

5. Certaines données concernant les États-Unis ont été recueillies par Huffman et Evenson (1993), NSF (2008), BEA (communiquées par Robbins et Moylan, 2007) et Pardey et Andersen (2009). Des principes directeurs sur la collecte de ces données ont été publiés par l'OCDE (2002 et 2005). Au sujet des données internationales, voir Pardey, Beintema, Dehmer et Wood (2006), ainsi que les indicateurs ASTI (Agricultural Science and Technology Indicators) sur le site web : http://www.asti.cgiar.org/.

6. S'agissant de la compilation de données relatives à la R-D en agriculture financée par le secteur privé aux États-Unis, voir Huffman et Evenson (1993), Klotz, Fuglie et Pray (1995), Fuglie et al. (1996), Echeverría et Byerlee (2002), et Dehmer, Pardey, et Alston (2009). Des informations limitées sont disponibles sur la recherche dans le secteur agricole du secteur privé dans d'autres pays. Voir par exemple Pardey, Beintema, Dehmer et Wood (2006). 
que l'accroissement mesuré de la productivité agricole est attribuable en totalité à la R-D (voire à une source particulière de R-D, comme la R-D publique d'un pays). De plus en plus, on se demande dans quelle mesure l'accroissement de la productivité pourrait être imputable à des facteurs autres que la R-D organisée, et notamment à l'évolution des conditions climatiques, aux changements institutionnels ou aux économies de taille associées à l'évolution structurelle de l'agriculture. Il s'agit dans une certaine mesure de questions ouvertes qu'il faudrait approfondir, mais dans bien des cas, il est probable que la recherche organisée soit le principal facteur de l'accroissement de la productivité observé et la question importante est celle de l'attribution entre les différentes sources de R-D.

\section{Aspects spatiaux du problème d'attribution de la recherche-développement}

L'attribution spatiale est importante car nous cherchons à faire correspondre des flux d'avantages à des flux de coûts, et la recherche agricole est financée principalement par des entités du secteur public qui sont définies au plan géopolitique. Qu'elles s'intéressent ou non aux retombées de la recherche agricole, les études imposent des hypothèses implicites ou explicites concernant leurs effets spatiaux compte tenu des frontières géopolitiques. Dernièrement, des agroéconomistes se sont efforcés de mieux tenir compte du fait que le savoir créé par une entité géopolitique particulière peut influer sur la technologie dans une autre région et avoir des conséquences importantes à la fois pour les créateurs des retombées externes et pour les bénéficiaires des retombées internes (voir Alston, 2002, pour une étude de ces publications et Alston et al., 2010, pour un examen plus récent axé sur les États-Unis).

De nombreux travaux ignorent purement et simplement les retombées, mais à partir de Griliches (1957), certaines études de l'adoption de technologies spécifiques ont tenu compte des retombées spatiales parmi les États et les régions d'un pays ${ }^{7}$. Plusieurs autres s'appuient sur les méthodes de régression pour évaluer l'ensemble des effets de la recherche agricole sur la productivité à l'aide de mesures de la R-D plus globales (à l'échelle de la région, de l'État ou du pays tout entier). Certaines d'entre elles tiennent compte des conséquences des retombées et concluent qu'elles sont importantes. Par exemple, aux États-Unis, Huffman et Evenson (1993) constatent qu'une part importante (45\% et plus) des avantages de la recherche menée dans les stations expérimentales agricoles des États (State Agricultural Experiment Stations) prend la forme de retombées inter-États. Cette mesure s'appuie sur la proximité spatiale. Selon Alston et al. (2010), la R-D conduite dans les différents États des États-Unis et la R-D relevant des autorités fédérales augmentent sensiblement et dans les mêmes proportions la productivité totale dans un État donné. Pour mesurer les retombées, ils s'appuient non pas sur la proximité spatiale, mais sur la similarité de l'éventail des productions entre différents États. Les choix de modélisation sont déterminés, au moins dans une certaine mesure, par les limites des données disponibles et par les exigences de parcimonie des modèles. La plupart des études des systèmes nationaux, indépendamment de la méthode employée, écartent

7. Par exemple, Evenson et Kislev (1973) ont analysé les retombées de la recherche sur le blé et le maïs, Araji, White et Guenther (1995) ont examiné les retombées de la recherche sur la pomme de terre et Maredia, Ward et Byerlee (1996) et Traxler et Byerlee (2001) ont fait des recherches sur les retombées concernant le blé. Pardey et al. (1996) ont analysé les effets aux États-Unis de la recherche variétale sur le riz et le blé conduite dans les centres internationaux de recherche des Philippines et du Mexique, et Pardey et al. (2006) ont évalué les retombées au Brésil de la recherche variétale internationale et institutionnelle. 
implicitement les retombées spatiales (d'après la méta-analyse présentée dans Alston et al., 2000a, moins de $20 \%$ des études prévoient la possibilité de retombées sous une forme ou une autre). Les études qui ne tiennent pas compte des retombées souffrent probablement d'un genre de biais de spécification.

\section{Aspects temporels du problème d'attribution de la $R-D$}

Il faut beaucoup de temps pour que la recherche exerce des effets sur la production et ces effets persistent ensuite pendant longtemps. Une partie du problème de l'attribution des fruits de la recherche consiste donc à mettre en lumière les caractéristiques du lien dynamique qui existe entre les dépenses de recherche, le stock de connaissances et la productivité. De nombreuses études antérieures s'appuient sur une analyse de régression dans laquelle une mesure de la production ou de la productivité agricole est fonction de variables représentatives de la recherche et de la vulgarisation agricoles, souvent dans le but d'estimer le taux de rentabilité de la recherche ${ }^{8}$. Il est essentiel de spécifier les déterminants de la relation temporelle entre les investissements dans la recherche et les variations de la production, laquelle dépend notamment de la dynamique de la création, de la dépréciation et de l'utilisation des connaissances. Seules quelques études exposent largement les justifications théoriques des modèles à retards échelonnés sur lesquels elles s'appuient pour modéliser la rentabilité de la recherche agricole.

Le tableau 1 résume certaines caractéristiques essentielles des modèles à retards échelonnés appliqués à la recherche dans les études de productivité agricole des pays de l'OCDE. Ce tableau est une version retravaillée du tableau 5 figurant dans Alston et al. (2000a). Jusqu'à ces derniers temps, il était courant de limiter la durée du décalage temporel à moins de 20 ans. Dans les premières études, les séries chronologiques disponibles étaient courtes et les durées des retards très courtes, mais dans les études plus récentes, les retards sont généralement plus longs. Dans la plupart des études, la représentation de la distribution des retards se limite à un petit nombre de paramètres, à la fois parce que la période couverte par les données n'est habituellement guère plus longue que la durée maximale supposée des retards et parce que les estimations des paramètres de retard sont instables et imprécises du fait de la forte colinéarité entre des séries multiples de dépenses passées de recherche 9 .

8. Alston et al. (2000a) donnent un compte rendu et une évaluation très complets de ces publications ; voir aussi Schuh et Tollini (1978), Norton et Davis (1981), Evenson (2002) et Alston, Andersen, James et Pardey (2010).

9. Comme l'explique Alston et al. (2000a), les types de structures des retards utilisés couramment pour construire un stock de recherche comprennent le V inversé de de Leeuw (Evenson, 1967, par exemple), le polynôme (par exemple, Davis 1980 ; Leiby et Adams, 2002 ; Thirtle et Bottomley, 1988) et le quadrilatère trapézoïdal (par exemple, Huffman et Evenson, 1989, 1992, 1993, 2006 ; Evenson, 1996). Un petit nombre d'études utilisent des retards de forme libre (par exemple, Ravenscraft et Scherer, 1982 ; Pardey et Craig, 1989 ; Chavas et Cox, 1992). 
Dans leur application qui utilise des séries longues de données au niveau des États concernant l'agriculture aux États-Unis, Alston et al. (2010) se prononcent en faveur d'une distribution des retards de type gamma impliquant une rémanence des effets de la recherche beaucoup plus longue que celle ressortant de la plupart des études précédentes - pour des raisons tant théoriques qu'économétriques ${ }^{10}$. Leurs calculs incitent à penser que les effets de la recherche s'étendent sur au moins 35 ans, voire 50 ans dans le cas des États-Unis, en passant par un point haut la $24^{\mathrm{e}}$ année ${ }^{11}$. Ce décalage relativement long a des conséquences tant pour les estimations économétriques des effets de la recherche sur la productivité que sur le taux implicite de rentabilité de la recherche.

Tableau 1. Structures des retards dans les études sur la recherche et la productivité agricole

\begin{tabular}{|c|c|c|c|c|c|c|}
\hline Caractéristique & $\begin{array}{c}\text { Nombre } \\
\text { d'estimations }\end{array}$ & 1958-69 & $1970-79$ & $1980-89$ & 1990-98 & $1958-98$ \\
\hline & Comptage & \multicolumn{5}{|c|}{ Pourcentage } \\
\hline \multicolumn{7}{|l|}{$\begin{array}{l}\text { Durée du retard } \\
\text { (avantages de la recherche) }\end{array}$} \\
\hline 0 à 10 ans & 253 & 9.7 & 6.2 & 17.9 & 12.7 & 13.4 \\
\hline 11 à 20 ans & 537 & 41.9 & 22.0 & 38.8 & 22.8 & 28.5 \\
\hline 21 à 30 ans & 376 & 0.0 & 20.7 & 12.0 & 25.9 & 19.9 \\
\hline 31 à 40 ans & 178 & 0.0 & 4.3 & 5.6 & 14.3 & 9.4 \\
\hline 40 jusqu'à $\infty$ ans & 141 & 0.0 & 9.5 & 6.6 & 7.6 & 7.5 \\
\hline$\infty$ années & 102 & 35.5 & 7.5 & 2.9 & 5.4 & 5.4 \\
\hline Non précisé ${ }^{1}$ & 109 & 12.9 & 13.1 & 3.2 & 4.9 & 5.8 \\
\hline Peu clair ${ }^{2}$ & 190 & 0.0 & 16.7 & 12.7 & 6.3 & 10.1 \\
\hline Total & 1886 & 100.0 & 100.0 & 100.0 & 100.0 & 100.0 \\
\hline
\end{tabular}

Ce tableau se fonde sur l'échantillon complet de 292 publications rendant compte de 1886 observations.

1. Les estimations non précisées sont celles pour lesquelles la durée des décalages entre recherche et retombées bénéfiques n'est pas indiquée explicitement.

2. La durée des décalages est peu claire.

Source : Alston et al. (2009b), adapté de Alston et al. (2000a).

10. Les arguments détaillés sont présentés dans Alston, Norton et Pardey (1995) et des éléments plus anciens sont présentés par Pardey et Craig (1988) et Alston, Craig et Pardey (1998). Voir aussi Huffman et Evenson (1989). Alston, Craig et Pardey (1998) examinent la question de la dépréciation des connaissances en se fondant sur des publications antérieures et ces arguments sont de nouveau présentés et affinés par Alston, Pardey et Ruttan (2008), et Alston, Andersen, James et Pardey (2010).

11. Alston, Pardey et Ruttan (2008) ont établi les retards dans l'adoption de technologies agricoles particulières et leurs résultats sont compatibles avec des retards généralement assez longs. 


\section{Résultats sur les conséquences économiques de la recherche-développement en agriculture}

Cette section présente un résumé très bref des éléments apportés par les travaux antérieurs sur la rentabilité de la R-D agricole au niveau mondial, d'après la méta-analyse de Alston et al. (2000a), et compare ces éléments à caractère général avec les résultats plus récents de Alston et al. (2010) pour les États-Unis. La section suivante propose d'autres interprétations des conséquences qui sont présentées brièvement ici. Le principal résultat à la fois de cette compilation au niveau mondial et des résultats récents portant sur les États-Unis est de montrer que le taux de rentabilité de la R-D agricole a généralement été très élevé, avec des taux moyens des avantages par rapport aux coûts bien supérieurs à 1.0. Les taux marginaux des avantages par rapport aux coûts se sont révélés supérieurs à 1.0 ce qui implique qu'il aurait été très profitable d'investir davantage. De ce point de vue, les résultats montrent de façon cohérente que les pays individuels et le monde dans son ensemble ont sous-investi dans la R-D agricole. A moins de penser que les avantages de la R-D agricole ont des taux de rentabilité fortement décroissant, et aucun fait ne nous amène à soutenir cette hypothèse, l'ampleur des taux marginaux des avantages par rapport aux coûts peut-être interprétée comme un signe de l'importance du sous-investissement.

\section{Synthèse des éléments apportés par les travaux antérieurs}

Alston et al. (2000a) ont mené à bien une méta-analyse très complète des études qui avaient fait état d'estimations de la rentabilité de la recherche-développement en agriculture. Relativement peu d'études ont été publiées dans la période qui a suivi, de sorte que cette analyse reste représentative. Comme le montre le rapport détaillé de la méta-analyse, les publications font état d'études réalisées dans de nombreux pays et s'appliquant à la R-D conduite dans beaucoup de pays également, stratifiées selon les caractéristiques des projets de recherche (domaine scientifique, produit agricole ou autre sujet, région géopolitique à laquelle l'étude s'applique, etc.) et selon différents aspects de la méthode d'analyse. Quelques points essentiels de la méta-analyse sont résumés ici et nous laissons le lecteur intéressé se reporter au rapport (Alston et al., 2000a) ou à l'article succinct (Alston et al., 2000b) pour de plus amples détails.

L'échantillon comprend 292 études rendant compte d'un total de 1852 estimations du taux de rentabilité de la R-D en agriculture, à partir desquelles Alston et al. (2000a) font état d'un taux interne de rentabilité global moyen de $81.3 \%$ avec un mode de $40 \%$ et une médiane de $44.3 \%$ (tableau 2). Après avoir laissé de côté quelques éléments atypiques et observations incomplètes, ils ont procédé à une analyse de régression en utilisant un échantillon de 1128 estimations avec une moyenne de $64.6 \%$, un mode de $28 \%$ et une médiane de $42.0 \%$. Ils ont trouvé des résultats qui étaient en général conformes aux attentes mais bien souvent, ils n'ont pas pu distinguer d'effets statistiquement significatifs sur les taux de rentabilité estimés liés à la nature des travaux évalués, au secteur auquel ils s'appliquaient ou à la méthode d'évaluation, parce que le ratio signal/bruit était trop faible. Toutefois, il ressort avec constance des études que le taux de rentabilité est élevé. La masse principale de la distribution des taux internes de rentabilité dont il est fait état dans les travaux antérieurs se situe entre 20 et $80 \%$ par an. D'autres examens des travaux antérieurs, qui ne portent pas nécessairement sur les mêmes études ou qui ne s'appuient pas systématiquement sur la même démarche, conduisent cependant à des conclusions générales similaires -par exemple Evenson (2002), et Fuglie et Heisey (2007). 
Alston et al. (2000a) parviennent à la conclusion que, selon les données disponibles, la R-D en agriculture est très bénéfique à la société. Cependant, ils font part d'un certain nombre de préoccupations quant aux méthodes utilisées dans les études, qui introduisent probablement des biais à la hausse dans les estimations. Ils estiment en particulier que de nombreuses études peuvent pâtir (a) de biais liés à l'utilisation, pour la recherche, de distributions de retards trop courtes (les résultats montrent qu'un allongement du décalage se traduit par une diminution des taux de rentabilité, ce qui est théoriquement prévisible), (b) de biais de "sélection", où seuls les investissements les plus performants dans la recherche sont soumis à l'évaluation (c) de biais d'attribution, faute de tenir compte de la contribution aux avantages mesurés des retombées imputables à d'autres organismes de recherche privés et publics tant dans la zone où ils sont implantés que dans d'autres États ou d'autres pays, ou (d) de biais dus à d'autres aspects des méthodes utilisées.

Tableau 2. Structures des retards et taux de rentabilité de la R-D en agriculture

\begin{tabular}{|c|c|c|c|c|c|c|c|}
\hline \multirow{2}{*}{ Caractéristique } & \multicolumn{2}{|c|}{ Estimations } & \multicolumn{5}{|c|}{ Taux de rentabilité } \\
\hline & Nombre & Part du total & Moyenne & Mode & Médiane & Minimum & Maximum \\
\hline & \multicolumn{2}{|c|}{ Comptage } & \multicolumn{5}{|c|}{ Pourcentage } \\
\hline 0 à 10 ans & 370 & 20.9 & 90.7 & 58.0 & 56.0 & -56.6 & 1219.0 \\
\hline 11 à 20 ans & 490 & 27.7 & 58.5 & 49.0 & 43.7 & -100.0 & 677.0 \\
\hline 21 à 30 ans & 358 & 20.2 & 152.4 & 57.0 & 53.9 & 0.0 & 5645.0 \\
\hline 31 à 40 ans & 152 & 8.6 & 64.0 & 40.0 & 41.1 & 0.0 & 384.4 \\
\hline 40 à $\infty$ ans & 113 & 6.4 & 29.3 & 20.0 & 19.0 & 0.3 & 301.0 \\
\hline$\infty$ années & 57 & 3.2 & 49.9 & 20.0 & 35.0 & -14.9 & 260.0 \\
\hline non précisé & 205 & 11.6 & 48.7 & 25.0 & 34.5 & 1.1 & 337.0 \\
\hline peu clair & 27 & 1.5 & 43.1 & 27 et 60 & 38.0 & 9.0 & 125.0 \\
\hline \multicolumn{8}{|l|}{$\begin{array}{l}\text { Temps de gestation de la } \\
\text { recherche }\end{array}$} \\
\hline inclus & 468 & 59.2 & 65.5 & 46.0 & 47.1 & -14.9 & 526.0 \\
\hline omis & 314 & 39.7 & 96.7 & 95.0 & 58.8 & 0.0 & 1219.0 \\
\hline non précisé ou peu clair & 8 & 1.0 & 25.1 & & 24.1 & 6.9 & 55.0 \\
\hline Total & 790 & 100.0 & 77.5 & 46 et 58 & 50.2 & -14.9 & 1219.0 \\
\hline \multicolumn{8}{|l|}{ Retombées } \\
\hline retombées internes & 291 & 16.7 & 94.5 & 95.0 & 68.0 & 0.0 & 729.7 \\
\hline retombées externes & 70 & 4.0 & 73.7 & 95.0 & 46.4 & 8.9 & 384.4 \\
\hline pas de retombées & 1428 & 81.7 & 78.8 & 49 et 57 & 40.0 & -100.0 & 5645.0 \\
\hline
\end{tabular}

Ce tableau s'appuie sur un échantillon de 292 publications au total, faisant état de 1886 observations. Pour toutes les caractéristiques, l'échantillon ne tient pas compte de deux points extrêmes et n'inclut que la rentabilité de la recherche, en associant recherche et vulgarisation, de sorte que la taille maximum de l'échantillon est de 1772 observations. Pour le délai de gestation de la recherche, l'échantillon ne comprend que les observations qui présentent un incontestable caractère décalé, ce qui se traduit par un échantillon de 790 observations. Pour les retombées, 25 observations ont été perdues car l'information était incomplète, d'où un échantillon de 1747 observations. Certaines estimations font état de retombées dans les deux sens.

Source : Rapporté par Alston et al. (2009b), à partir des données présentées dans Alston et al. (2000a). 


\section{Éléments récents sur la $R-D$ en agriculture aux États-Unis}

Plus récemment, Alston, Andersen, James et Pardey (2010) ont modélisé la productivité agricole des États des États-Unis sur la période 1949-2002, en tant que fonction des investissements publics dans la recherche et la vulgarisation agricoles sur la période 1890-2002. Dans cette étude, une attention particulière a été portée aux questions méthodologiques soulevées dans Alston et al. (2000a), en particulier concernant la modélisation de la distribution des retards et les retombées des résultats de la recherche entre États. Les retombées entre États sont représentées par un indicateur de la proximité technologique fondé sur les corrélations entre combinaisons de produits. La distribution des retards a été estimée à l'aide d'un modèle de distribution souple de type gamma. Les résultats tendaient à confirmer des décalages relativement longs (un délai d'une durée totale de 50 ans avec un point haut la $24^{\mathrm{e}}$ année, mais un impact en grande partie épuisé en l'espace de 40 ans) avec une part très importante de l'accroissement de la productivité d'un État attribuable à la recherche menée par d'autres États et par l'État fédéral. Autrement dit, les avantages nationaux des investissements d'un État dans la recherche dépassent largement les avantages qu'en tire cet État lui-même, ce qui constitue une source supplémentaire de dysfonctionnements du marché de la R-D en agriculture puisque les autorités d'un État ne tiennent généralement pas compte des retombées bénéfiques dans les autres États ou tout au moins les sous-estiment (largement).

Le tableau 3 est une synthèse des résultats du modèle le plus utilisé par les auteurs. Relatif aux États-Unis, il montre la distribution des avantages des investissements des États et de l'État fédéral dans la recherche et la vulgarisation agricoles. Il indique les avantages obtenus d'une part dans l'État lui-même et d'autre part au niveau national, sous forme de ratios avantages-coûts et de taux internes de rentabilité ${ }^{12}$. Il en ressort qu'une augmentation marginale des investissements dans la recherche et la vulgarisation agricoles des 48 États contigus des États-Unis génère des avantages pour chaque État d'une valeur se situant entre 2 USD et 58 USD par dollar investi, et à 21 USD en moyenne par État (les ratios avantages-coûts les plus bas concernent généralement les États où le secteur agricole est modeste et en recul, notamment en Nouvelle-Angleterre). Si l'on tient compte des retombées dans d'autres États, les investissements des différents États dans la recherche agricole génèrent des avantages au niveau national d'une valeur se situant entre 10 USD et 70 USD par dollar investi et à 32 USD en moyenne par État. Comparable, le ratio marginal avantages-coûts de la recherche interne du ministère de l'Agriculture des États-Unis est à 18 USD par dollar investi dans la recherche.

Les ratios avantages-coûts du tableau 3 sont généralement élevés et pourraient sembler invraisemblables à certains lecteurs. Pourtant, si l'on se reporte aux résultats généraux figurant dans les publications dépouillées par Alston et al. (2000a), résumés dans le tableau 2 et analysés par d'autres auteurs (par exemple Evenson, 2002; Fuglie et Heisey, 2007), ils concordent avec les taux internes de rentabilité dans le bas de la fourchette. En particulier, les estimations des taux «individuels » de rentabilité au niveau d'un État se situent entre 7.4 et $27.6 \%$ avec une moyenne de $18.9 \%$ par an et par État, et les estimations des taux «collectifs » de rentabilité au niveau national se situent entre 15.3 et $29.1 \%$ avec une moyenne de $22.9 \%$ par an et par État. Le taux de rentabilité de la

12. Alston, Andersen, James et Pardey (2010) expliquent de manière convaincante les raisons de rendre compte des ratios avantages-coûts plutôt que des taux internes de rentabilité dans ce cas. Des taux internes de rentabilité sont donnés ici pour faciliter les comparaisons avec d'autres études. 
recherche interne du ministère de l'Agriculture des États-Unis est quant à lui de $18.7 \%$ par an.

Ces observations confirment la suggestion de Alston et Pardey (2001), à savoir que si l'on accorde plus d'attention à l'attribution temporelle et spatiale, on a des chances d'aboutir à des estimations plus basses des ratios avantages-coûts de la R-D en agriculture (ou des taux internes de rentabilité correspondants). Toutefois, même en tenant compte de possibles erreurs de mesure et de biais, les données montrent que la recherche agricole génère de très gros dividendes. Cela conforte l'opinion selon laquelle, d'une part, l'agriculture se caractérise par des dysfonctionnements du marché dus au fait que les inventions sont insuffisamment protégées par les droits de propriété, et d'autre part, en dépit d'une intervention importante des pouvoirs publics pour corriger ces dysfonctionnements, les pays continuent de sous-investir dans la recherche agricole.

Tableau 3. Ratios avantages-coûts et taux internes de rentabilité de la R-D en agriculture aux États-Unis

\begin{tabular}{|c|c|c|c|c|}
\hline \multirow[t]{2}{*}{ Bénéfices } & \multicolumn{2}{|c|}{$\begin{array}{l}\text { Ratio avantages-coûts } \\
\text { (taux réel } \\
\text { d'actualisation de } 3 \% \text { ) }\end{array}$} & \multicolumn{2}{|c|}{$\begin{array}{l}\text { Taux interne } \\
\text { de rentabilité }\end{array}$} \\
\hline & $\begin{array}{l}\text { Dans l'État } \\
\text { lui-même }\end{array}$ & $\begin{array}{c}\text { Au niveau } \\
\text { national }\end{array}$ & $\begin{array}{l}\text { Dans l'État } \\
\text { lui-même }\end{array}$ & $\begin{array}{c}\text { Au niveau } \\
\text { national }\end{array}$ \\
\hline & \multicolumn{2}{|c|}{ Ratio } & \multicolumn{2}{|c|}{ Pourcentage annuel } \\
\hline \multicolumn{5}{|l|}{$\begin{array}{l}\text { Recherche et vulgarisation } \\
\text { au niveau de l'État } \\
48 \text { États }\end{array}$} \\
\hline Moyenne & 21.0 & 32.1 & 18.9 & 22.7 \\
\hline Minimum & 2.4 & 9.9 & 7.4 & 15.3 \\
\hline Maximum & 57.8 & 69.2 & 27.6 & 29.1 \\
\hline \multicolumn{5}{|l|}{ Quelques États } \\
\hline Californie & 33.3 & 43.4 & 24.1 & 26.1 \\
\hline Minnesota & 40.6 & 55.4 & 24.7 & 27.3 \\
\hline Wyoming & 12.7 & 23.6 & 16.8 & 20.9 \\
\hline \multicolumn{5}{|l|}{ Régions } \\
\hline Pacifique & 21.8 & 32.9 & 20.2 & 23.5 \\
\hline Montagnes & 20.0 & 31.6 & 19.0 & 22.7 \\
\hline Plaines du nord & 42.4 & 54.5 & 24.9 & 27.0 \\
\hline Plaines du sud & 20.2 & 31.0 & 19.5 & 22.7 \\
\hline Centre & 33.7 & 46.8 & 23.1 & 25.9 \\
\hline Sud-est & 15.1 & 26.7 & 17.6 & 22.0 \\
\hline Nord-est & 9.4 & 18.4 & 14.0 & 19.0 \\
\hline $\begin{array}{l}\text { Recherche au ministère de } \\
\text { l'Agriculture des États-Unis }\end{array}$ & & 17.5 & & 18.7 \\
\hline
\end{tabular}

Source : Alston et al. (2010). 


\section{Interprétation des résultats}

Alston et al. (2010) ont montré que leurs estimations spécifiques des ratios avantagescoûts étaient quelque peu sensibles aux choix de modélisation, mais que les résultats généraux étaient déterminés par des paramètres fondamentaux. Précisément, la valeur annuelle des gains de productivité agricole équivaut plusieurs fois à celle des dépenses de recherche. Par voie de conséquence, les avantages de l'accroissement de la productivité attribué à la R-D en agriculture sont au moins dix fois plus importants que les coûts, indépendamment des méthodes de mesure ou des hypothèses concernant l'attribution (par exemple la forme et la durée de la distribution des retards, les retombées interrégionales ou interinstitutionnelles, ou les rôles de la R-D ou de la vulgarisation du secteur privé). Cet aspect caractérise les résultats dans toutes les publications et non pas seulement dans Alston et al. (2010).

\section{Approximation simple}

Pour faire la démonstration de ce point, Alston et al. (2010) ont examiné la valeur des gains de productivité depuis 1949 par rapport à la valeur des investissements dans la recherche auxquels ces gains sont comparés. Aux États-Unis, l'augmentation de la productivité globale des facteurs dans l'agriculture a été estimée à $1.79 \%$ par an sur la période 1949-2002. Si l'on applique ce taux de croissance de $1.79 \%$ par an sur 53 ans, l'indice de productivité passe de 100 en 1950 à environ 258 en 2002. Autrement dit, si l'on considère que les quantités d'intrants utilisées ont été maintenues au niveau de 1949 sur toute la période, la production augmente dans la proportion 2.6:1. De ce fait, sur la production totale de 2002, d'une valeur de 174.1 milliards USD, seulement $39 \%$, soit 67.3 milliards USD, peuvent être attribués aux intrants traditionnels fondés sur les technologies de 1949, et les 106.9 milliards USD restants sont imputables aux facteurs qui ont été à l'origine de l'accroissement de la productivité entretemps. Parmi ces facteurs, il faut citer les nouvelles technologies, développées et adoptées grâce à la recherche et à la vulgarisation agricoles.

Le chiffre de 106.9 milliards USD se rapporte aux avantages d'une seule année, 2002, liés aux gains de productivité depuis 1949. Alston et al. (2010) ont calculé les valeurs correspondantes pour chacune des années sur la période 1949-2002 en divisant la valeur réelle de la production agricole (a) en une partie attribuable aux intrants réels avec la productivité de 1949, et (b) un chiffre résiduel attribuable à l'accroissement de la productivité depuis 1949. Ce flux de valeurs résiduelles a été exprimé en dollars constants de 2000, en divisant les valeurs nominales par le déflateur du PNB. En appliquant un taux d'actualisation réel de $3 \%$ par an, on obtient un flux de valeurs résiduelles équivalant à un montant global de 7335 milliards USD en 2002, qui représente le bénéfice énorme retiré de l'accroissement de la productivité agricole aux États-Unis durant la période postérieure à la Deuxième Guerre mondiale. Le flux d'avantages découlant de l'accroissement de la productivité est imputable à plusieurs facteurs, comme les investissements publics et privés dans la recherche et la vulgarisation agricoles aux ÉtatsUnis et ailleurs, l'amélioration des infrastructures, les investissements dans l'éducation et le perfectionnement du capital humain, ainsi que les retombées des connaissances et de la technologie en provenance d'autres secteurs (non agricoles).

La valeur actualisée des dépenses de recherche et de vulgarisation sur la période 1949-2002 était de 326 milliards USD. Le ratio des avantages totaux par rapport à ces 
coûts est de 22.5. Cependant, ce ratio brut avantages-coûts ne tient pas compte des décalages temporels entre la recherche et ses applications - ce qui signifie que les avantages des premières années reflètent les dépenses de recherche réalisées avant 1949 , tandis que les dépenses de recherche des dernières années produiront des avantages après 2002 - et attribue tous les gains de productivité à la recherche publique. On peut obtenir d'autres estimations en incluant les dépenses de recherche des années précédentes et en faisant en sorte qu'une fraction de la totalité des avantages ne soit pas attribuable à la recherche publique. Par exemple, si nous faisons en sorte que la moitié des avantages soit attribuable à d'autres sources, le ratio avantages-coûts est diminué de moitié et passe à 12/1. De même, si nous prenons en compte les dépenses de recherche réalisées à partir de 1910 et comparons les avantages sur la période 1949-2002 avec les coûts sur la période 1910-2002, le ratio avantages-coûts tombe alors à 17/1.

\section{Représentation analytique de l'approximation simple}

Ces mesures brutes présentent un avantage au sens où elles évitent les complications de l'estimation économétrique, mais elles nécessitent des hypothèses adaptées en ce qui concerne la proportion des avantages attribuable à la recherche et à la vulgarisation agricoles, et la correspondance entre dépenses de recherche et période de flux d'avantages. Mais surtout, elles donnent des estimations sur une même échelle que celles qui sont établies au moyen de méthodes économétriques plus complexes et moins transparentes, et elles montrent que les estimations des ratios avantages-coûts sont déterminées par des relations fondamentales entre les taux d'accroissement de la productivité et leur valeur cumulée, comparée aux montants correspondants des dépenses annuelles de R-D en agriculture.

On peut utiliser un modèle mathématique relativement simple pour démontrer ces liens et pour montrer comment étendre les résultats généraux à d'autres contextes que celui des États-Unis, par exemple à d'autres pays de l'OCDE où les taux d'accroissement de la productivité et l'intensité de la recherche agricole ont été similaires à ceux des États-Unis. Considérons un scénario dans lequel l'action publique prévoit de consacrer chaque année un montant réel fixe $R$ aux dépenses de R-D en agriculture, et ce à perpétuité. La valeur actualisée des coûts de cet investissement dans la recherche est égale à :

(1) $\quad P V C_{t}=\sum_{n=0}^{\infty} R(1+r)^{-n}=\frac{R}{r}$

Après un retard de $L$ années, ce flux d'investissements dans la recherche contribue à un taux annuel composé d'accroissement de la productivité agricole de $g$ à perpétuité. Pour toute année future, les avantages sont égaux à la différence entre la valeur réelle de la production, compte tenu de l'accroissement de la productivité, et la valeur en l'absence d'accroissement de la productivité. Par souci de simplicité, supposons qu'en l'absence de ces dépenses de recherche, la valeur réelle de la production agricole soit constante à $V$ par an (c'est-à-dire $V_{t+n}=V$ pour toutes les valeurs de $n$ ). Alors, si $n \geq L$ :

$$
B_{t+n}=\left((1+g)^{n-L}-1\right) V
$$


La valeur actualisée de ce flux d'avantages est égale à :

$$
P V B_{t}=\sum_{n=0}^{\infty} B_{t+n}(1+r)^{-n}=\sum_{n=L}^{\infty}\left((1+g)^{n-L}+1\right) V(1+r)^{-n}=(1+r)^{-L} \frac{V}{r}\left(\frac{g}{r-g}\right)
$$

Ce résultat s'applique aussi longtemps que $r>g$, sinon il n'est pas défini. Dans la réalité, cette condition est probablement respectée car $g$ est le taux de croissance de la productivité attribuée aux investissements dans la recherche, qui est généralement bien en-deçà de 2-3\% par an, tandis que $r$ est le taux d'actualisation approprié, généralement compris entre 3 et $5 \%$.

Si nous définissons le ratio avantages-coûts, $B C R$, comme étant égal à la valeur actualisée des avantages divisée par la valeur actualisée des coûts,

$$
B C R=\frac{P V B_{t}}{P V C_{t}}=\frac{(1+r)^{-L} \frac{V}{r}\left(\frac{g}{r-g}\right)}{\frac{R}{r}}=(1+r)^{-L} \frac{g}{(r-g)}\left(\frac{V}{R}\right)
$$

Supposons que nous utilisions des valeurs plausibles $r=0.05$ (soit un taux d'intérêt réel de $5 \%$ par an), $L=10$ ans, $g=0.02$ (soit un taux composé d'accroissement de la productivité de $2 \%$ par an attribuable à la R-D), et $R / V=25$ (correspondant à un coefficient d'intensité de la recherche agricole $i=0.04$ ). Avec ces valeurs, le facteur d'actualisation est $(1+r)^{-L}=0.61$, et $B C R=10.2$.

Le tableau 4 présente les ratios avantages-coûts correspondants pour une série de valeurs des paramètres de l'équation du $B C R$. On peut voir que le ratio avantages-coûts est directement proportionnel à l'inverse du coefficient d'intensité de la recherche (c'està-dire inversement proportionnel au rapport entre les dépenses de recherche et la valeur de la production), de telle sorte que le doublement de l'intensité de la recherche divise par deux le ratio avantages-coûts. De même, les ratios avantages-coûts sont sensibles à d'autres paramètres. En particulier, si le taux annuel d'accroissement de la productivité associé à une intensité de recherche donnée est ramené de $2 \%$ (dans la moitié supérieure du tableau) à $1 \%$ (dans la moitié inférieure du tableau), les ratios avantages-coûts sont réduits de bien plus de la moitié ; si le taux d'actualisation passe de $3 \%$ à $5 \%$ par an, là encore les ratios avantages-coûts sont réduits de plus de moitié ; et si le décalage entre recherche et retombées est doublé, passant de 10 à 20 ans, les ratios avantages-coûts sont réduits d'environ un tiers. Si l'on conjugue ces variations, la combinaison la plus favorable de paramètres aboutit à un ratio avantages-coûts qui représente environ 30 fois le ratio avantages-coûts découlant de la combinaison la moins favorable. La combinaison la moins favorable est toutefois relativement extrême, ce qui ne l'empêche pas pour autant d'être assortie d'un ratio avantages-coûts nettement supérieur à un. 
Tableau 4. Ratios approximatifs avantages-coûts découlant d'une série de paramètres

\begin{tabular}{|c|c|c|c|c|}
\hline \multirow[b]{2}{*}{$\begin{array}{c}\text { Inverse du } \\
\text { coefficient } \\
\text { d'intensité de } \\
\text { recherche, } R / V\end{array}$} & \multicolumn{2}{|c|}{ Taux d'actualisation, $r=3 \%$} & \multicolumn{2}{|c|}{ Taux d'actualisation, $r=5 \%$} \\
\hline & $\begin{array}{l}\text { Décalage entre } \\
\text { recherche et } \\
\text { retombées } \\
L=10 \text { ans }\end{array}$ & $\begin{array}{c}\text { Décalage entre } \\
\text { recherche et } \\
\text { retombées } \\
L=20 \text { ans }\end{array}$ & $\begin{array}{l}\text { Décalage entre } \\
\text { recherche et } \\
\text { retombées } \\
L=10 \text { ans }\end{array}$ & $\begin{array}{l}\text { Décalage entre } \\
\text { recherche et retombées } \\
\qquad=20 \text { ans }\end{array}$ \\
\hline & \multicolumn{4}{|c|}{ Accroissement de la productivité $g=2 \%$ par an } \\
\hline 50 (i = $2 \%)$ & 74.4 & 55.4 & 20.5 & 12.6 \\
\hline \multirow[t]{2}{*}{25 (i = 4 \%) } & 37.2 & 27.7 & 10.2 & 6.3 \\
\hline & \multicolumn{4}{|c|}{ Accroissement de la productivité $g=1 \%$ par an } \\
\hline 50 (i = $2 \%)$ & 18.6 & 13.8 & 7.7 & 4.7 \\
\hline 25 (i = $4 \%)$ & 9.3 & 6.9 & 3.8 & 2.3 \\
\hline
\end{tabular}

Source : Élaboré par l'auteur à partir des formules figurant dans le texte.

\section{Conclusion}

Les publications sur l'économie de la R-D en agriculture sont abondantes. Ce rapport met l'accent sur certains domaines essentiels où les résultats peuvent être fragiles ou biaisés en raison des choix de modélisation faits par les économistes. La création des «données » utilisées dans nos analyses est une étape délicate. Dans la mesure où ces données conditionnent souvent directement l'interprétation des résultats, il incombe à leur utilisateur de faire le nécessaire au moins pour déterminer comment elles ont été conçues, mais il n'existe pas de mécanisme pour l'y contraindre et il ne semble pas que ce travail fasse l'objet d'efforts particuliers.

Parallèlement aux données, les modèles utilisés pour mesurer les avantages de la recherche s'améliorent au fil des ans. L'analyse révèle certains domaines où les résultats sont sensibles aux choix de modélisation, notamment la représentation du changement technologique dans le modèle, le traitement des retombées et la distribution des retards. Il s'agit essentiellement de problèmes économétriques qui sont souvent difficiles à résoudre avec les données disponibles, mais auxquels il faut apporter une solution car ils peuvent avoir des répercussions importantes sur les résultats. La question de la spécification du changement technique imputable à la recherche dans les modèles reste en grande partie sans réponse. Davantage de progrès ont été faits en ce qui concerne les retards et les retombées. Dans les études qui s'intéressent à ces deux aspects, les retombées sont en général de plus en plus importantes et les retards de plus en plus longs. Les modèles qui ne représentent pas correctement les retombées ou qui tronquent les retards attribuent généralement à la recherche des taux de rentabilité plus élevés. On a pu constater que d'autres choix de spécification, comme les modalités de prise en compte des distorsions du marché dues à la puissance commerciale des entreprises, à l'action publique ou aux externalités environnementales, avaient des effets relativement importants sur les estimations de la distribution des avantages et relativement peu d'incidences sur les estimations des avantages totaux. 
Les économistes ont accumulé beaucoup d'éléments convaincants qui démontrent que le monde dans son ensemble, et les différents pays, retirent d'énormes avantages de l'accroissement de la productivité de l'agriculture rendu possible, en grande partie, par l'évolution technologique découlant des investissements publics et privés dans la R-D en agriculture. Selon les données, ces avantages sont plusieurs fois supérieurs aux coûts. Il en est ainsi même si nous minorons considérablement les estimations au motif que nous soupçonnons qu'elles peuvent être biaisées à la hausse, peut-être par inadvertance, en raison de choix méthodologiques malencontreux ou de limitations des données disponibles du même ordre que celles que nous avons examinées dans la présente étude. Comme les taux marginaux des avantages par rapport aux coûts ont été bien supérieurs à 1.0, il aurait été profitable d'investir davantage dans la R-D agricole, et cela probablement dans une large mesure. Nous pouvons en déduire, entre autres, que malgré l'intervention importante des pouvoirs publics, le monde a systématiquement sous-investi dans la R-D en agriculture et va probablement continuer à le faire. 


\section{Références}

Acquaye. A.K.A., J.M. Alston. et P.G. Pardey (2000), "A Disaggregated Perspective on Post-War Productivity Growth in U.S. Agriculture: Isn't that Spatial?" presented at the NC-208 Conference on Agricultural Productivity: Data. Methods. and Measures, Waugh Auditorium, USDA-ERS, Washington D.C., 9-10 mars.

Acquaye. A.K.A., J.M. Alston. et P.G. Pardey (2003), "Post-War Productivity Patterns in U.S. Agriculture: Influences of Aggregation Procedures in a State-Level Analysis", American Journal of Agricultural Economics, vol 85, $\mathrm{n}^{\mathrm{O}} 1$ (février), pp. 59-80.

Ahearn. M., J. Yee, V. E. Ball. et R. Nehring (1998), "Agricultural Productivity in the United States", Agricultural Information Bulletin $\mathrm{n}^{\circ} 740$. Economic Research Service. U.S. Department of Agriculture (Ministère de l'Agriculture des États-Unis).

Akino. M. et Y. Hayami (1975), "Efficiency and Equity in Public Research: Rice Breeding in Japan's Economic Development", American Journal of Agricultural Economic, vol 57, n ${ }^{\circ} 1$, pp. 1-10.

Alston. J.M. (2002), "Spillovers", Australian Journal of Agricultural and Resource Economics, vol 46, n⿳⺈ 3 (septembre), pp. 315-346.

Alston. J.M. (2007), "Benefits and Beneficiaries from U.S. Farm Subsidies", AEI Policy Series: Agricultural Policy for 2007 Farm Bill and Beyond. Washington DC: American Enterprise Institute.

2007.

http://aic.ucdavis.edu/research/farmbill07/aeibriefs/20070515_alstonSubsidiesfinal.pdf

Alston. J.M., M.A. Andersen, J.S. James. et P.G. Pardey (2009), "The Economics of Agricultural R\&D", Annual Review of Resource Economics, vol. 1. (octobre), pp 15-35.

Alston. J.M., M.A. Andersen, J.S. James et P.G. Pardey (2010), Persistence Pays: U.S. Agricultural Productivity Growth and the Benefits from Public R\&D Spending, New York, Springer.

Alston. J.M., B.A. Babcock. et P.G. Pardey eds (2010), The Shifting Patterns of Agricultural Productivity Worldwide, CARD-MATRIC Electronic Book, Ames Iowa, Center for Agricultural and Rural Development. http://www.matric.iastate.edu/shifting_patterns

Alston. J.M., J.M. Beddow. et P.G. Pardey (2008), “Agricultural Research. Productivity. and Food Commodity Prices", ARE Update novembre-décembre. http://www.agecon.ucdavis.edu/extension/update/articles/v12n2_5.pdf

Alston. J.M., J.M. Beddow. et P.G. Pardey (2009), "Mendel versus Malthus: Research. Productivity. and Food Prices in the Long Run", InSTePP Working Paper, St Paul, University of Minnesota, janvier.

Alston. J.M., C. Chan-Kang, M.C. Marra, P.G. Pardey. et T.J. Wyatt (2000a), A Meta Analysis of Rates of Return to Agricultural R\&D: Ex Pede Herculem?, Washington D.C., IFPRI (Institut international de recherche sur les politiques alimentaires) Research Report , $\mathrm{n}^{\circ} 113$.

Alston. J.M., B.J. Craig et P.G. Pardey (1998), "Dynamics in the Creation and Depreciation of Knowledge. and the Returns to Research", EPTD Discussion Paper $n^{\circ} 35$, Washington D.C., International Food Policy Research Institute, août. 
Alston. J.M., G.W. Edwards et J.W. Freebairn (1988), "Market Distortions and the Benefits from Research", American Journal of Agricultural Economics, vol 70, nº 2 (mai), pp. 281-288.

Alston. J.M., M.C. Marra, P.G. Pardey. et T.J. Wyatt (2000b), "Research Returns Redux: A Meta Analysis of the Returns to Agricultural R\&D", Australian Journal of Agricultural and Resource Economics, vol 44, $\mathrm{n}^{\circ}$ 2, pp. 185-215.

Alston. J.M. et W.J. Martin (1995), "Reversal of Fortune: Immiserizing Technological Change in Agriculture", American Journal of Agricultural Economics , vol 77 (mai), pp. 251-259.

Alston. J.M., G.W. Norton. et P.G. Pardey (1995), Science Under Scarcity: Principles and Practice for Agricultural Research Evaluation and Priority Setting, Ithaca, Cornell University Press, 1995 (réimprimé sous couverture brochée par CAB International 1998).

Alston. J.M. et P.G. Pardey (1993), "Market Distortions and Technological Progress in Agriculture", Technological Forecasting and Social Change, vol 43, n ${ }^{0} 3 / 4$ (mai/juin), pp. 301319.

Alston. J.M. et P.G. Pardey (1996), Making Science Pay: The Economics of Agricultural R\&D Policy. Washington D.C.: American Enterprise Institute Press.

Alston. J.M., P.G. Pardey. et V.H. Smith eds (1999), Paying for Agricultural Productivity. Baltimore: Johns Hopkins University Press.

Alston. J.M. et P.G. Pardey (2001), "Attribution and Other Problems in Assessing the Returns to Agricultural R\&D", Agricultural Economics, vol 25, n 2-3(septembre), pp. 141-152.

Alston. J.M. et P.G. Pardey (2006), "Farm Productivity and Inputs" sub-chapter comprising an essay and 16 data tables. including 210 series. in S. Carter, S. Gartner, M. Haines, A. Olmstead, R. Sutch et G. Wright. éd. Historical Statistics of the United States-Millennial Edition, Cambridge, Cambridge University Press.

Alston. J.M., P.G. Pardey et V.W. Ruttan (2008), "Research Lags Revisited: Concepts and Evidence from U.S. Agriculture." Paper presented at the 2008 Economic History Association meetings, New Haven CT, septembre 12-14.

Andersen. M.A. (2005), Pro-cyclical Productivity Patterns in U.S. Agriculture. Thèse de doctorat non publiée, Davis, University of California.

Andersen. M.A., P.G. Pardey, B.J. Craig et J.M. Alston (2009), "Measuring Capital Inputs Using the Physical Inventory Method: with Application to U.S. Agriculture. , inSTePP Working Paper, St Paul, University of Minnesota.

Antle. J.M. et P.L. Pingali (1994), "Pesticides. Productivity, and Farmer Health: A Philippines Case Study", American Journal of Agricultural Economics , vol 76 (août), pp. 418-430.

Araji. A.A., F.C. White, F.C., et J.F. Guenthner (1995), "Spillovers and the Returns to Agricultural Research for Potatoes", Journal of Agricultural and Resource Economics, vol 20n 2 (décembre), pp. 263-76.

Banque mondiale (2007), Rapport sur le développement dans le monde 2008: L'agriculture au service $d u$ développement, Washington D.C., Banque mondiale, octobre. http://siteresources.worldbank.org/INTRDM2008INFRE/Resources/French-version-WDR2008-July-7.pdf

Ball. V.E. (1985),“Output. Input. and Productivity Measurement in U.S. Agriculture.” American Journal of Economics, vol 67, $\mathrm{n}^{\circ} 3$ (août), pp. 475-86.

Ball. V.E. (1994), "Measuring Agricultural Productivity in U.S. Agriculture", Proceedings of the NC-208 symposium at Orlando. Florida. March 1993. in W.B. Sundquist (ed.) Evaluating Research and Productivity in an Era of Resource Scarcity. Staff paper P94-2, Department of Agricultural and Applied Economics, University of Minnesota, Février. 
Ball V.E., J.C. Bureau, R. Nehring. et A. Somwaru (1997), "Agricultural Productivity Revisited", American Journal of Agricultural Economics, vol 79, n 4 (novembre), pp. 1045-63.

Ball V.E., F.M. Gollop, A. Kelly-Hawke, et G.P. Swinand (1999), "Patterns of State Productivity Growth in the U.S. Farm Sector: Linking State and Aggregate Models", American Journal of Agricultural Economics, vol 81, n 1 (février), pp. 164-179.

Ball V.E., et R. Nehring (1998), "Patterns of State Productivity Growth in the U.S. Farm Sector", Economic Research Service staff paper No. 9804, Washington D.C., U.S. Department of Agriculture.

Barton. G.T. et M.R. Cooper (1948), "Relation of Agricultural Production to Inputs", Review of Economics and Statistics, vol 2, pp. 117-126.

Barton. G.T. et D.D. Durost (1960), "The New USDA Index of Inputs", Journal of Farm Economics, vol 42, $\mathrm{n}^{\mathrm{o}}$ 5, pp. 1398-1410.

Baum. W.C. (1986), Partners Against Hunger: The Consultative Group for International Agricultural Research. (Groupe consultatif pour la recherche agricole internationale) (GCRAI), Washington D.C., Banque mondiale

Bouchet. F., D. Orden, G.W. Norton (1989), "Sources of Growth in French Agriculture", American Journal of Agricultural Economics. 71(2), pp. 280-293.

Brennan. J.P. (2007), "Beyond Semi-Dwarf Wheat Yield Increases: Impacts on the Australian Wheat Industry of On-Going Spillovers from the International Maize and Wheat Improvement Center", Australian Journal of Agricultural and Resource Economics, vol 51, n 4 (décembre), pp. 385-401.

Byerlee. D. et G. Traxler (2001), "The Role of Technology Spillovers and Economies of Size in the Efficient Design of Agricultural Research Systems", Chapter 9 in J. M. Alston. P. G. Pardey. et M. J. Taylor, éd. Agricultural Science Policy: Changing Global Agendas, Baltimore, Johns Hopkins University Press.

Capalbo. S.M. et T.T. Vo. (1988), "A Review of the Evidence on Agricultural Productivity and Aggregate Technology", Chapter 3 in S. M. Capalbo. et J. M. Antle. éd.,Agricultural Productivity: Measurement and Explanation, Washington D.C., Resources for the Future.

Chavas. J.-P. et T.L. Cox (1992), "A Nonparametric Analysis of the Effects of Research on Agricultural Productivity", American Journal of Agricultural Economics, vol 74(août), pp. 583591.

Craig. B.J. et P.G. Pardey (1990a),“Multidimensional Output Indices", Department of Agricultural and Applied Economics Staff Paper P90-63, St. Paul, University of Minnesota, octobre.

Craig. B.J. et P.G. Pardey (1990b), "Patterns of Agricultural Development in the United States", Department of Agricultural and Applied Economics Staff Paper P90-72, St. Paul, University of Minnesota, décembre.

Craig. B.J. et P.G. Pardey (2001), "Input. Output. and Productivity Developments in U.S. Agriculture", Chapter 5 in J.M. Alston, P.G. Pardey et M. J. Taylor éd. Agricultural Science Policy: Changing Global Agendas, Baltimore, Johns Hopkins University Press.

Davis. J.S. (1980), "A Note on the Use of Alternative Lag Structures for Research Expenditure in Aggregate Production Function Models", Revue canadienne d'agroéconomie, vol 28, pp. 7276.

Davis. G.C. et M.C. Espinoza (1998), “A Unified Approach to Sensitivity Analysis in Equilibrium Displacement Models", American Journal of Agricultural Economics, vol 80, pp. 868-79.

Dehmer, S., P.G. Pardey. et J.M. Alston (2009), "The Shifting Structure of Private Food and Agricultural R\&D in the United States Since the 1950s", inSTePP Working Paper, St Paul, University of Minnesota. 
Duncan. R.C. (1972), "Evaluating Returns to Research in Pasture Improvement", Australian Journal of Agricultural Economics, vol 16, n 3, pp. 153-168.

Duncan. R.C.. et C. Tisdell (1971), "Research and Technical Progress: The Returns to the Producers", Economic Record, vol 47, n 117, pp. 124-129.

Echeverría. R.G. et D. Byerlee (éd.) (2002), Agricultural Research Policy in an Era of Privatization. Wallingford. U.K.: CAB International.

Evenson. R.E.(1967), "The Contribution of Agricultural Research to Production", Journal of Farm Economics, vol 49 (décembre), pp. 1415-1425.

Evenson. R.E. (1996), "Two Blades of Grass: Research for U.S. Agriculture", Chapter 11 in J.M. Antle et D.A. Sumner (éd..), The Economics of Agriculture Volume 2, Papers in Honor of D. Gale Johnson, Chicago, University of Chicago Press, pp. 171-203.

Evenson. R.E. (2002), “Economic Impacts of Agricultural Research and Extension.” Chapter 11 in B.L. Gardner et G.C. Rausser eds. Handbook of Agricultural Economics. Volume 1A: Agricultural Production, New York, Elsevier.

Evenson. R.E. et Kislev. Y. (1973), "Research and Productivity in Wheat and Maize", Journal of Political Economy, vol 81, pp. 1309-1329.

Evenson. R.E. et Y. Kislev (1975), Agricultural Research and Productivity, New Haven, Yale University Press.

Evenson. R.E., D. Landau et D. Ballou (1987), “Agricultural Productivity Measures for US States 1950-82", In W.B. Sundquist (ed.) Evaluating Agricultural Research and Productivity, University of Minnesota, Miscellaneous Publication 52-1987.

Frisvold. G.B. (1997), "Multimarket Effects of Agricultural Research with Technological Spillovers", In T.W. Hertel (Ed.) Global Trade Analysis, New York, Cambridge University Press.

Frisvold. G., J. Sullivan et A. Raneses (1999), "Who Gains from Genetic Improvement in U.S. Crops", AgBioForum, vol 2, n 3\&4, pp. 237-246.

Freebairn. J.W. (1992), "Evaluating the Level and Distribution of Benefits from Dairy Industry Research", Australian Journal of Agricultural Economics, vol 36, n 2, pp. 141-166.

Fuglie. K.O. et P.W. Heisey (2007), Economic Returns to Public Agricultural Research. USDA. ERS Economic Brief $\mathrm{n}^{\mathrm{o}} 10$, Washington D.C., USDA, septembre.

Fuglie. K.O., N. Ballenger, K. Day, C. Klotz, M. Ollinger, J. Reilly, U. Vasavada et J. Yee (1996), Agricultural Research and Development: Public and Private Investments Under Alternative Markets and Institutions, Agricultural Economics Report No. 735, Washington D.C., USDA, mai.

Gardner. B.L. (1992), "How the Data We Make Can Unmake Us: Annals of Factology", American Journal of Agricultural Economics, vol 74, n 5 (décembre), pp. 1066-1075.

Griliches. Z. (1957), "Hybrid Corn: An Exploration in the Economics of Technological Change", Econometrica, vol 25, pp. 501-522.

Griliches. Z. (1958), "Research Costs and Social Returns: Hybrid Corn and Related Innovations", Journal of Political Economy, vol 66, n 5, pp. 419-431.

Griliches. Z. (1960), "Measuring Inputs in Agriculture: A Critical Survey", Journal of Farm Economics, vol 42, n' 5 , pp. 1411-1427.

Griliches. Z. (1964), "Research Expenditures. Education and the Aggregate Agricultural Production Function", American Economic Review, vol 54, nº 6, pp. 961-974. 
Griliches. Z. (1979), "Issues in Assessing the Contribution of R\&D to Productivity Growth", Bell Journal of Economics, vol 10, $\mathrm{n}^{\circ}$ 1, pp. 92-116.

Griliches. Z. (1994), "Productivity. R\&D. and the Data Constraint", American Economic Review, vol 84, nº 1 (mars), pp. 1-23.

Griliches. Z. (1996), "R\&D and Productivity: The Unfinished Business", Chapter in Z. Griliches $R \& D$ and Productivity: The Econometric Evidence, Chicago, Chicago University Press.

Griliches. Z. (2001), "R\&D and Productivity: The Unfinished Business", Chapter 3 in J.M. Alston, P.G. Pardey et M. J. Taylor (éd.), Agricultural Science Policy: Changing Global Agendas, Baltimore, Johns Hopkins University Press.

Harberger. A.C. (1971), “Three Basic Postulates for Applied Welfare Economics: An Interpretive Essay", Journal of Economic Literature, vol 9, n 3, pp. 785-797.

Hayami. Y. et V.W. Ruttan (1971), Agricultural Development: An International Perspective, Baltimore, Johns Hopkins University Press, réimprimé en 1985.

Huffman. W.E. et R.E. Evenson (1989), "Supply and Demand Functions for Multiproduct U.S. Cash Grain Farms: Biases Caused by Research and other Policies", American Journal of Agricultural Economics, vol 71 (août), pp. 761-773.

Huffman. W.E. et R.E. Evenson (1992), "Contributions of Public and Private Science and Technology to U.S. Agricultural Productivity", American Journal of Agricultural Economics, vol 74 (août), pp 752-756.

Huffman. W.E. et R.E. Evenson (1993), Science for Agriculture: A Long-Term Perspective, Ames, Iowa State University Press.

Huffman. W.E. et R.E. Evenson (2006), Science for Agriculture: A Long-Term Perspective, Oxford, Blackwell Publishing, deuxième édition.

Huffman. W.E. et R.E. Evenson (2001), "Structural and Productivity Change in US Agriculture. 1950-1982", Agricultural Economics, vol 24, nº 2 (janvier), pp. 127-147.

Huffman. W.E. et R.E. Evenson (2006), "Do Formula or Competitive Grant Funds have Greater Impacts on State Agricultural Productivity", American Journal of Agricultural Economics, vol 88, $\mathrm{n}^{\circ} 4$, pp. 783-98.

Huffman. W.E. et R.E. Just (1994), "Funding. Structure. and Management of Public Agricultural Research in the United States", American Journal of Agricultural Economics, vol 76, pp. 744 759 .

Jaffe. A.B. (1986), "Technological Opportunity and Spillovers of R\&D: Evidence from Firm's Patents. Profits. and Market Value", American Economic Review, vol 76, n 5 (décembre), pp. 984-1001.

Jorgenson. D.W. et F.M. Gollop (1992), "Productivity Growth in U.S. Agriculture: A Postwar Perspective", American Journal of Agricultural Economics, vol 74, $\mathrm{n}^{\mathrm{o}} 3$ (août), pp. 745-750.

Just. R.E., J.M. Alston et D. Zilberman. éd. (2006), Regulating Agricultural Biotechnology: Economics and Policy, NewYork, Springer-Verlag.

Klotz. C., K. Fuglie et C. Pray (1995), Private Sector Agricultural Research Expenditures in the United States: 1960-1992, ERS Staff Paper $\mathrm{n}^{\circ}$ 9525, Washington D.C., United States Department of Agriculture, octobre.

Lapan. H. et G. Moschini (2004), "Innovation and Trade with Endogenous Market Failure: The Case of Genetically Modified Crops", American Journal of Agricultural Economics , vol 86, n 3 (août), pp 634-638. 
Leiby. J.D. et G.D. Adams (2002), "The Returns to Agricultural Research in Maine: The Case of a Small Northeastern Experiment Station", Northeastern Journal of Agricultural and Resource Economics, vol 20, pp. 1-14.

Lindner. R.K. et F.G. Jarrett (1978), "Supply Shifts and the Size of Research Benefits", American Journal of Agricultural Economics, vol 60, n 1, pp. 48-58.

Lindner. R.K. et F.G. Jarrett (1980), "Supply Shifts and the Size of Research Benefits: Reply", American Journal of Agricultural Economics , vol 62, nº 4, pp. 841-844.

Loomis R.A. et G.T. Barton (1961), Productivity of Agriculture: United States. 1870-1958.. USDA Technical Bulletin, $\mathrm{n}^{\circ}$ 1238. Washington D.C.: U.S. Department of Agriculture, avril.

Lynam. J.K. et P.G. Jones (1984), "Benefits of Technical Change as Measured by Supply Shifts: An Integration of Theory and Practice", mimeo, CIAT, Colombia, février.

Moschini. G. et H. Lapan (1997), "Intellectual Property Rights and the Welfare Effects of Agricultural R\&D", American Journal of Agricultural Economics, vol 79 (novembre), pp. 1229-1242.

NSF (2008), National Science Board. Science and Engineering Indicators 2008 (two volumes), Arlington, VA, National Science Foundation.

OCDE (2003), Manuel de Frascati 2002: Méthode type proposée pour les enquêtes sur la recherche et le développement expérimental, OCDE, Paris.

OCDE (2005), Manuel d'Oslo : Principes directeurs pour le recueil et l'interprétation des données sur l'innovation, $3^{\text {ème }}$ édition, OCDE, Paris.

Oehmke. J.F. et E.W. Crawford (2002), "The Sensitivity of Returns to Research Calculations to Supply Elasticity", American Journal of Agricultural Economics, vol 64, n² 2, pp. 366-369.

Pachico, D. J. Lynam et P.G. Jones (1987), "The Distribution of Benefits from Technical Change among Classes of Consumers and Producers: An Ex Ante Analysis of Beans in Brazil", Research Policy, vol 16, pp. 279-285.

Pardey. P.G., J.M. Alston. et R.R. Piggott. éd. (2006), Agricultural R\&D in the Developing World: Too Little. Too Late?, Washington D.C., International Food Policy Research Institute.

Pardey. P.G., J.M. Alston. et V.W. Ruttan (2010), "The Economics of Innovation and Technical Change in Agriculture", in B.H. Hall and N. Rosenberg éd., The Handbook of Economics of Technical Change, Amsterdam, Elsevier.

Pardey. P.G. et M.A. Andersen (2009), "A Long-Run Price Index and the Real Cost of U.S. Agricultural Research", InSTePP Working Paper, St Paul, University of Minnesota.

Pardey. P.G., N.M. Beintema, S. Dehmer et S. Wood (2006), Agricultural Research: A Growing Global Divide?, IFPRI Food Policy Report, Washington. D.C., Institut international de recherche sur les politiques alimentaires.

Pardey. P.G. et B. Craig (1989), "Causal Relationships Between Public Sector Agricultural Research Expenditures and Output", American Journal of Agricultural Economics, vol 71 (février), pp. 9-19.

Pardey. P.G., B.J. Craig. et K.W. Deininger (1994), “A New Look at State-Level Productivity Growth in U.S. Agriculture." Proceedings of the NC-208 Symposium at Orlando. Florida. March 1993, in W.B. Sundquist (éd.) Evaluating Research and Productivity in an Era of Resource Scarcity, Staff paper P94-2, Department of Agricultural and Applied Economics, University of Minnesota, février.

Petersen. W.L. (1967), "Returns to Investment in Poultry Research in the United States", Journal of Farm Economics, vol 49, n³ 3 pp. 656-670. 
Ravenscraft. D. et F.M. Scherer (1982), "The Lag Structure of Returns to Research and Development", Applied Economics, vol 14, pp. 603-620.

Robbins. C.A. et C.E. Moylan (2007), Research and Development Satellite Account Update: Estimates for 1959-2004, Washington D.C., Bureau of Economic Analysis. Consulté en novembre 2007 à l'adresse suivante http://www.bea.gov/scb/pdf/2007/10\%200ctober/1007_rd text.pdf

Ruttan. V.W. (1982), Agricultural Research Policy, Minneapolis, University of Minnesota Press.

Schultz. T.W. (1953), The Economic Organization of Agriculture, New York, McGraw-Hill.

Schultz. T.W. (1978), "On Economics and Politics of Agriculture", Chapter in T. W. Schultz. ed. Distortions in Agricultural Incentives, Bloomington, Indiana University Press.

Schuh. G.E. et H. Tollini (1979), Costs and Benefits of Agricultural Research: State of the Arts, World Bank Staff Work Paper $n^{0} 360$, Washington D.C, Banque mondiale.

Scobie. G.M. (1976), "Who Benefits from Agricultural Research?", Review of Marketing and Agricultural Economics, vol 44, n 4, pp. 197-202.

Thirtle. C.G. et P. Bottomley (1988), "Is Publicly Funded Agricultural Research Excessive?", Journal of Agricultural Economics, vol 31, pp. 99-111.

Tintner. G. (1944), "A Note on the Derivation of Production Functions from Farm Records", Econometrica, vol 1, pp. 26-34.

Traxler. G. et D. Byerlee. (2001), "Linking Technical Change to Research Effort: An Examination of Aggregation and Spillovers Effects", Agricultural Economics, vol 24, pp. 235-246.

Falck-Zepeda, J.B., G. Traxler et R.G. Nelson (2000), "Surplus Distribution from the Introduction of a Biotechnology Innovation", American Journal of Agricultural Economics, vol 82, n 2 , pp. 360-69.

Voon. J.P. et G.W. Edwards (1991), "The Calculation of Research Benefits with Linear and Nonlinear Specifications of Demand and Supply Functions", American Journal of Agricultural Economics, vol 74, n 3, pp. 415-420.

Wohlgenant. M.K (1997), "The Nature of the Research-Induced Supply Shift", Australian Journal of Agricultural and Resource Economics, vol 41, n 3 , pp. 385-400.

Zhao. X., W. E. Griffiths, G.R. Griffith et J.D. Mullen (2000), "Probability Distributions for Economic Surplus Changes: The Case of Technical Change in the Australian Wool Industry", Australian Journal of Agricultural and Resource Economics, vol 44, pp. 83-106. 